\title{
A General Easy-to-use Expression for Uncertainty Evaluation in Residual Voltage Measurement
}

\author{
${ }^{1}$ Alessandro Mingotti, ${ }^{2}$ Annalisa Baldi, ${ }^{1}$ Lorenzo Peretto, ${ }^{1}$ Roberto Tinarelli \\ ${ }^{1}$ Department of Electrical, Electronic and Information Engineering "G. Marconi”, ${ }^{2}$ Department of Mathematics \\ Alma Mater Studiorum - University of Bologna \\ Bologna, Italy
}

\begin{abstract}
The paper addresses one of the new and most important issues arising when Low Power Voltage Transformers (LPVTs) are used in power network substations for evaluating, among others, the residual voltage measurement. Conversely to open-triangle inductive instrument transformers, the use of phase voltage transformers for measuring the residual voltage gets challenging due to the very high accuracy required for the three LPVTs. In the paper, a general expression to estimate the residual voltage measurement uncertainty, starting from the LPVTs accuracy, is presented. The effectiveness of the proposed approach is then confirmed with both Monte Carlo simulations and actual measurements on a general three-phase system.
\end{abstract}

Keywords - Voltage Transformer; Residual Voltage; Simulation; Low Power Instrument Transformer; Power Transformer; Accuracy Class; Uncertainty

\section{INTRODUCTION}

The decarbonisation of energy systems has been a political decision taken in the recent years by all most industrialized countries in the world. European Commission's energy and climate policy, such as the SET-Plan, foresees that the context of the future scenarios for electric power networks will be that of ensuring a stable and secure power supply as Renewable Energy Sources (RES) penetration increases up to $100 \%$.

As a matter of fact, the wide-scale introduction of decentralized RES is causing significant and unprecedented changes in electrical power grids. Consequently, future electrical power grids will require real-time capable control and monitoring systems to ensure stability under increasingly complex and challenging conditions [1].

The generation of analogue measurement and control systems in power grid substations are approaching the end of their useful lifespan. More often their replacement is based on digital substation automation solutions according to IEC 61850 [2] and the use of new technologies to perform in a more efficient way (like the massive use of Ring Main Units), measurements and control of the power network operation [3].

More specifically, Instrument Transformers (ITs) [4-6] are experiencing special attention by Utilities and private customers for different important reasons. Among the paramount ones, with the event of Smart Grids and Distributed Energy Resources (DER) new performance and features are requested to the IT in order to accomplish real-time network control with the highest efficiency, speed and accuracy [7]. For instance, the large use of power inverters for interconnecting large photovoltaic plants to

This research has been partially funded by the European project Horizon H2020 "SOGNO: Service Oriented Grid for the Network of the Future". Project no. 774613

Annalisa Baldi is supported by MAnET Marie Curie Initial Training Network, by GNAMPA of INdAM, Italy, and by PRIN of the MIUR, Italy. the grid has led to the injection of high order harmonics, which can interfere with industrial frequency component or among them to give rise to intermodulation. All this, needs that such spectral components be correctly and accurately measured in order to let such systems run under real-time feedback control. Moreover, in case of off-nominal frequency, protection relays must now trip faster than before (in few ms, instead of tens of $\mathrm{ms}$ as in the past). Furthermore, given that energy is no more flowing in just one direction (multiple producers or prosumers are now interconnected to the same grid), very accurate energy and power measurements must be performed. This, to correctly split the energy production revenues among prosumers and for a proper accurate reactive energy injection into the grid. Again, the mass deployment of secondary substations and measurement nodes have also yield to have space and size constraints. Therefore, new requests for reduced dimensions of all electrical apparatus and systems have become a key parameter. Last but not least, the development and diffusion of digital communications between different nodes of the power network have requested for the ITs, as stated before, to feature also digital outputs [8].

In light of all this, one of the most important measurements for network stability and diagnostic still remains the residual voltage. Its measurement is mandatory for protection coordination, like the implementation of differential protections, to correctly classify ground faults, etc. Normally, such a measurement was and is presently performed by using inductive voltage transformers star-connected at the primary side and with open triangle at the secondary side. Typical accuracies required for the residual voltage measurements are in the order of some percent, while residual voltage values are in the order of few percent (4\%) [9].

In case the residual voltage is evaluated by using the line voltages (sum of the three ITs secondary voltages), reaching such an accuracy in its measurement gets very challenging. In particular, in the case of RES, where the power flow becomes bi-directional and the nodes voltages might suffer significant changes. Moreover, this is even worse if the ITs are not working at ambient temperature, as demonstrated in literature [10]. This is what is requested to the new devices used as voltage sensors, the Low Power Voltage Transformers (LPVT). This new kind of transformers have lower outputs (voltage in the order of few volt and a maximum power typically equal or lower than $1 \mathrm{VA}$ ) with respect to the traditional ones. Usually such devices reduce the phase-to-ground primary voltage to amplitudes of few volts. 
The secondary voltage is referred to ground as well. So, especially for LPVTs, the accuracy requirements for the residual voltage measurements need to be guaranteed. This results in the adoption of LPVTs with high accuracy classes (far better than those of the inductive voltage transformers).

The paper is aimed to present a novel study developed from the results of the related paper [11] to correlate the uncertainty affecting the measurement of the residual voltage with the accuracy class of the LPVTs.

First of all, a new easy-to-use expression for estimating the uncertainty on the residual voltage, for a generic 3-phase system, is derived. It will be shown that it provides accurate results without the use of complex and long calculation as it would be required by the application of analytical or numerical methods suggested by the Guide to the expression of Uncertainty in Measurement (GUM) [12] as well as its Supplement 1 [13].

Such a study is requested by industry and might be used for completing two important Standards: the IEC 61869-11 [14] on LPVTs, and the future IEC 61869-105 [15] document dealing with uncertainty in calibration of ITs. Moreover, it will result useful to power network and system designers and operators for selecting suitable LPVTs according also to the accuracy requested for the residual voltage measurement.

As for the proposed expression, its validity has been confirmed with both computer simulation and actual measurement. Tests are performed with a measurement setup developed for the specific purpose of the residual voltage measurement.

The paper is structured as follows: in Section II the backbone concepts of [11] are recalled and the expression of the residual voltage is presented in the case of a generic (balanced or unbalanced) 3-phase system. Section III briefly summarize the uncertainty results obtained in [11] and provides the residual voltage uncertainty for the aforementioned case-study. In Section IV, the setup proposed for the residual voltage measurement is described. Tests and results of the performed measurements are presented in Section V. Finally, Section VI summarize the presented study along with some conclusions.

\section{ResiduAl Voltage}

\section{A. Case-study definition}

According to the International Electrotechnical Vocabulary (IEV) the residual voltage $v_{r}(t)$ is defined as "the sum of the instantaneous values of all three line-to-earth voltages, in a three-phase system" [16]:

$$
v_{r}(t)=v_{1}(t)+v_{2}(t)+v_{3}(t)
$$

where $v_{1}(t), v_{2}(t)$ and $v_{3}(t)$ are the instantaneous line-to-earth voltages of line 1,2 and 3 , respectively. In the case of a sinusoidal steady-state condition (1) turns into:

$$
\bar{V}_{R}=\bar{V}_{1}+\bar{V}_{2}+\bar{V}_{3}
$$

where $\bar{V}_{i}$ is the phasor of the generic quantity $v_{i}(t)$. Either if the residual voltage is a phasor (as in (2)) or a waveform (as in (1)), in practical application only its module is used.

Fig. 1 shows a typical setup for the measurement of such a quantity, referred to as $\bar{V}_{R}$. It consists in three LPVTs and an Intelligent Electronic Device (IED), which acquires the LPVTs outputs and computes the residual voltage. Therefore, the value attributed to $\bar{V}_{R}$ is affected by the effect of the uncertainty sources (gain and non-linearity error, offset, noise, ratio and phase error, etc.) located in the LPVTs as well as in the IED. Usually, the latter can be considered negligible with respect to former one $[3,18]$.

\section{B. Mathematic development}

In light of this, let us consider three LPVTs featuring ratio errors $\varepsilon_{1}, \varepsilon_{2}$ and $\varepsilon_{3}$ and phase errors $\Delta \varphi_{1}, \Delta \varphi_{2}$, and $\Delta \varphi_{3}$ as defined in [6]. Therefore, starting from (2), the residual voltage, $\bar{V}_{R}$ can be expressed as a function of such uncertainty contributions:

$$
\begin{gathered}
\bar{V}_{R}=\left[V_{1}\left(1+\varepsilon_{1}\right) e^{j\left(\vartheta_{1}+\Delta \varphi_{1}\right)}+V_{2}\left(1+\varepsilon_{2}\right) e^{j\left(\vartheta_{2}+\Delta \varphi_{2}\right)}+\right. \\
\left.V_{3}\left(1+\varepsilon_{3}\right) e^{j\left(\vartheta_{3}+\Delta \varphi_{3}\right)}\right]
\end{gathered}
$$

where the $V_{i}$ and $\vartheta_{i}$ are the generic RMS value and phase angle of the related phasor $\bar{V}_{i}$, respectively.

For the sake of simplicity but without loss of generality in [11], as a first scenario, a balanced three-phase system condition has been assumed. This means that:

$$
V_{1}=V_{2}=V_{3}=V
$$

and

$$
\vartheta_{1}=0, \vartheta_{2}=\frac{2}{3} \pi \text { and } \vartheta_{3}=-\frac{2}{3} \pi \text {. }
$$

Such assumption led (see [11] for details) to the following expression of the residual voltage module:

$$
\begin{gathered}
\left|\bar{V}_{R}\right|=V\left[\left(\varepsilon_{1}-\frac{\sqrt{3}}{2} \Delta \varphi_{2}-\frac{1}{2} \varepsilon_{2}+\frac{\sqrt{3}}{2} \Delta \varphi_{3}-\frac{1}{2} \varepsilon_{3}\right)^{2}+\right. \\
\left.\left(\Delta \varphi_{1}-\frac{1}{2} \Delta \varphi_{2}+\frac{\sqrt{3}}{2} \varepsilon_{2}-\frac{1}{2} \Delta \varphi_{3}-\frac{\sqrt{3}}{2} \varepsilon_{3}\right)^{2}\right]^{1 / 2}
\end{gathered}
$$

It can be noted that when all $\varepsilon_{i}$ and $\Delta \varphi_{i}$ are zero, (6) provides $\left|\bar{V}_{R}\right|=0$, according to the assumption of balanced voltages. Therefore, the expression between square brackets represents the error on $\bar{V}_{R}$ when a balanced three-phase system of amplitude $V$ is considered. Of course, such error can be computed only if the values of the accuracy parameters $\varepsilon$ and $\Delta \varphi$ are already known, for each VT. Otherwise, (6) can be also used to evaluate the uncertainty on $\bar{V}_{R}$ if $\varepsilon_{i}$ and $\Delta \varphi_{i}$ are treated as random variables and one of the methods, suggested by GUM [11] and its supplement 1 [13], is applied.

In this paper, a general expression of $\left|\bar{V}_{R}\right|$ is derived from (3), no matter the system is balanced or unbalanced. Hence, by considering the Euler formulae (3) turns into:

$$
\begin{gathered}
\bar{V}_{R}=\left\{V_{1}\left[\left(1+\varepsilon_{1}\right)\left(\cos \left(\vartheta_{1}+\Delta \varphi_{1}\right)+\mathrm{j} \sin \left(\vartheta_{1}+\Delta \varphi_{1}\right)\right)\right]+\right. \\
V_{2}\left[\left(1+\varepsilon_{2}\right)\left(\cos \left(\vartheta_{2}+\Delta \varphi_{2}\right)+\mathrm{j} \sin \left(\vartheta_{2}+\Delta \varphi_{2}\right)\right)\right]+ \\
\left.V_{3}\left[\left(1+\varepsilon_{3}\right)\left(\cos \left(\vartheta_{3}+\Delta \varphi_{3}\right)+\mathrm{j} \sin \left(\vartheta_{3}+\Delta \varphi_{3}\right)\right)\right]\right\}
\end{gathered}
$$

By defining three parameters helpful to increase the reader comprehension:

$$
\begin{gathered}
L=\cos \left(\vartheta_{1}+\Delta \varphi_{1}\right)+\mathrm{j} \sin \left(\vartheta_{1}+\Delta \varphi_{1}\right) \\
M=\cos \left(\vartheta_{2}+\Delta \varphi_{2}\right)+\mathrm{j} \sin \left(\vartheta_{2}+\Delta \varphi_{2}\right) \\
\mathrm{N}=\cos \left(\vartheta_{3}+\Delta \varphi_{3}\right)+\mathrm{j} \sin \left(\vartheta_{3}+\Delta \varphi_{3}\right)
\end{gathered}
$$

(7) can be re-written as: 


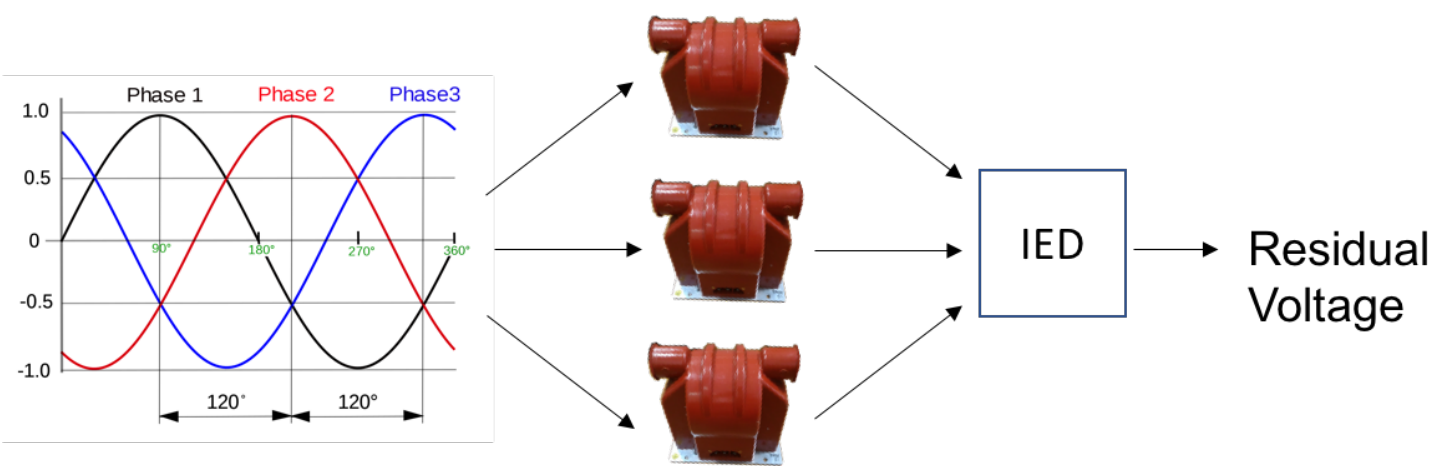

Fig. 1. Schematic of a typical setup for the measurement of three-phase system of symmetric voltages

$$
\bar{V}_{R}=V_{1} L+\varepsilon_{1} V_{1} L+V_{2} M+\varepsilon_{2} V_{2} M+V_{3} N+\varepsilon_{3} V_{3} N,
$$

which highlights the terms not affected by the ratio error of the LPVTs. Focusing on these terms $\left(V_{1} L, V_{2} M\right.$ and $\left.V_{3} N\right)$, in particular on the first, and by applying the addition sine formulae:

$$
\begin{gathered}
V_{1} L=V_{1}\left[\cos \left(\vartheta_{1}\right) \cos \left(\Delta \varphi_{1}\right)-\sin \left(\vartheta_{1}\right) \sin \left(\Delta \varphi_{1}\right)\right]+j V_{1} \\
{\left[\sin \left(\vartheta_{1}\right) \cos \left(\Delta \varphi_{1}\right)+\cos \left(\vartheta_{1}\right) \sin \left(\Delta \varphi_{1}\right)\right] .}
\end{gathered}
$$

Given that, in actual conditions, $\Delta \varphi_{i}$ is small, $\cos \left(\Delta \varphi_{i}\right) \cong 1$ and $\sin \left(\Delta \varphi_{i}\right) \cong \Delta \varphi_{i}$ is assumed; hence:

$$
V_{1} L=\bar{V}_{R(1)}+V_{1} \Delta \varphi_{1}\left[\operatorname{jicos}\left(\vartheta_{1}\right)-\sin \left(\vartheta_{1}\right)\right]
$$

where $\bar{V}_{R(1)}$ is the term of the residual voltage, included in (10), not affected by the ratio and phase error of the LPVTs:

$$
\bar{V}_{R(1)}=V_{1} \cos \left(\vartheta_{1}\right)+j V_{1} \sin \left(\vartheta_{1}\right) \text {. }
$$

Therefore, by writing all terms of (9) as it has been done in (11) for the term $V_{1} L$, and by neglecting all the second order terms (i.e. those which are a product of LPVT parameters $\varepsilon_{i}$ and $\Delta \varphi_{i}$ ), (9) becomes:

$$
\begin{gathered}
\bar{V}_{R}=\bar{V}_{R(1)}\left(1+\varepsilon_{1}\right)+\bar{V}_{R(2)}\left(1+\varepsilon_{2}\right)+\bar{V}_{R(3)}\left(1+\varepsilon_{3}\right)+ \\
V_{1} \Delta \varphi_{1}\left[j \cos \left(\vartheta_{1}\right)-\sin \left(\vartheta_{1}\right)\right]+V_{2} \Delta \varphi_{2}\left[j \cos \left(\vartheta_{2}\right)-\sin \left(\vartheta_{2}\right)\right] \\
+V_{3} \Delta \varphi_{3}\left[j \cos \left(\vartheta_{3}\right)-\sin \left(\vartheta_{3}\right)\right],
\end{gathered}
$$

which represents the residual voltage general expression in the case of an unbalanced 3-phase system. Eq. (13) can be expressed in terms of module (14), real (15) and imaginary part (16):

$$
\begin{gathered}
\left|\bar{V}_{R}\right|=\sqrt{\left(\operatorname{Re}\left[\bar{V}_{R}\right]\right)^{2}+\left(\operatorname{Im}\left[\bar{V}_{R}\right]\right)^{2}} \\
\operatorname{Re}\left[\bar{V}_{R}\right]=V_{1} \cos \left(\vartheta_{1}\right)\left(1+\varepsilon_{1}\right)+V_{2} \cos \left(\vartheta_{2}\right)\left(1+\varepsilon_{2}\right)+ \\
V_{3} \cos \left(\vartheta_{3}\right)\left(1+\varepsilon_{3}\right)-V_{1} \sin \left(\vartheta_{1}\right) \Delta \varphi_{1}-V_{2} \sin \left(\vartheta_{2}\right) \Delta \varphi_{2}- \\
V_{3} \sin \left(\vartheta_{3}\right) \Delta \varphi_{3} \\
\operatorname{Im}\left[\bar{V}_{R}\right]=V_{1} \sin \left(\vartheta_{1}\right)\left(1+\varepsilon_{1}\right)+V_{2} \sin \left(\vartheta_{2}\right)\left(1+\varepsilon_{2}\right)+ \\
V_{3} \sin \left(\vartheta_{3}\right)\left(1+\varepsilon_{3}\right)+V_{1} \cos \left(\vartheta_{1}\right) \Delta \varphi_{1}+V_{2} \cos \left(\vartheta_{2}\right) \Delta \varphi_{2}+ \\
V_{3} \cos \left(\vartheta_{3}\right) \Delta \varphi_{3} .
\end{gathered}
$$

Of course, if all parameters $\varepsilon_{i}$ and $\Delta \varphi_{i}$ are equal to zero, (14) provides $\left|\bar{V}_{R}\right|=0$ as for the case of a balanced 3-phase system. To summarise the overall procedure, (15) and (16) have been obtained by applying two simple and common assumptions: to consider $\Delta \varphi_{i}$ a small angle and to neglect terms which are the product of two LPVT parameters (for example $\Delta \varphi_{i} * \varepsilon_{i}$ ). As for the first assumption, errors of $2 \cdot 10^{-5}$ on the cosine value and 3 $\mu \mathrm{rad}$ on the angle one are made if $\Delta \varphi_{i}=6 \mathrm{mrad}$ is taken (limit for the 0.5 accuracy class, worst case). For the latter assumption instead, this turns into considering zero in place of $10^{-5}$ (worst case for the 0.5 accuracy class).

\section{UNCERTAINTY EVALUATION}

As mentioned in Section II, the residual voltage error can be obtained only if the values of the accuracy parameters $\varepsilon$ and $\Delta \varphi$ are already known for each VT. However, in practical situations, this is not possible and the uncertainty affecting $\left|\bar{V}_{R}\right|$ must be determined. In this connection, parameters $\varepsilon$ and $\Delta \varphi$ are treated as random variables. The GUM and its Supplement 1 provide detailed explanation on how estimating such value, but the implementation of the methods they provided may be quite complex and not easy for technicians who are not metrologist or university professors. For this reason, in light of the experience gained by the authors on the topic [18] and considering the existing literature [19], two simplified expressions for the uncertainty evaluation of the residual voltage measurement are presented. The first one is recalled from [11] and can be applied when an (almost) balanced three-phase system is concerned. The second one is a new expression developed by the authors to be used with whatever 3-phase system. The aim is to provide an easy-to-use formula directly applicable in field, when only the accuracy class of the LPVTs is known.

\section{A. Balanced 3-phase system}

In [11], (6) has been re-written in terms of 2 new random variables $X$ and $Y$ :

$$
\begin{aligned}
& X=\varepsilon_{1}-\frac{\sqrt{3}}{2} \Delta \varphi_{2}-\frac{1}{2} \varepsilon_{2}+\frac{\sqrt{3}}{2} \Delta \varphi_{3}-\frac{1}{2} \varepsilon_{3} \\
& Y=\Delta \varphi_{1}-\frac{1}{2} \Delta \varphi_{2}+\frac{\sqrt{3}}{2} \varepsilon_{2}-\frac{1}{2} \Delta \varphi_{3}-\frac{\sqrt{3}}{2} \varepsilon_{3},
\end{aligned}
$$

whose variances $\sigma_{X}^{2}$ and $\sigma_{Y}^{2}$, pending that the 3 LPVTs have the same accuracy class, are:

$$
\sigma_{X}^{2}=\frac{3}{2}\left(\sigma_{\Delta \varphi}^{2}+\sigma_{\varepsilon}^{2}\right)
$$


Then, it is:

$$
\sigma_{Y}^{2}=\frac{3}{2}\left(\sigma_{\Delta \varphi}^{2}+\sigma_{\varepsilon}^{2}\right)
$$

$$
\sigma_{X}^{2}=\sigma_{Y}^{2}
$$

In (19) and (20) $\sigma_{\varepsilon}^{2}$ and $\sigma_{\Delta \varphi}^{2}$ are the variances of the random variables $\varepsilon$ and $\Delta \varphi$ representing the chosen accuracy class for the LPVTs. Therefore, the probability distribution associated to the random variable $\left|\bar{V}_{R}\right|$ defined by (6), is a well-known Rayleigh one [11]. Hence, the variance $\sigma_{R}^{2}$ of $\left|\bar{V}_{R}\right|$ is:

$$
\sigma_{R}^{2}=\left(2-\frac{\pi}{2}\right) \sigma^{2},
$$

where $\sigma^{2}=\sigma_{X}^{2}=\sigma_{Y}^{2}$.

\section{B. Generic 3-phase system}

The balanced 3-phase condition cannot always be met. Hence, an easy-to-use expression applicable in all cases is required. To this purpose, let us start from (15) and (16). Both are a linear combination of six zero-mean random variables $\varepsilon_{1}$, $\varepsilon_{2}, \varepsilon_{3}, \Delta \varphi_{1}, \Delta \varphi_{2}$ and $\Delta \varphi_{3}$. It is well known that, given a random variable $f$ defined as:

$$
f=a g+b h
$$

where $g$ and $h$ are generic independent random variables and $a$ and $b$ are numerical coefficients, its variance $\sigma_{f}^{2}$ is given by:

$$
\sigma_{f}^{2}=a^{2} \sigma_{g}^{2}+b^{2} \sigma_{h}^{2}
$$

where $\sigma_{g}^{2}$ and $\sigma_{h}^{2}$ are the variances of $g$ and $h$, respectively.

Therefore, by applying (24) to (15) and (16):

$$
\begin{array}{r}
\sigma_{U}^{2}=V_{1}{ }^{2} \cos ^{2}\left(\vartheta_{1}\right) \sigma_{\varepsilon 1}^{2}+V_{2}{ }^{2} \cos ^{2}\left(\vartheta_{2}\right) \sigma_{\varepsilon 2}^{2}+V_{3}{ }^{2} \cos ^{2}\left(\vartheta_{3}\right) \sigma_{\varepsilon 3}^{2}+ \\
V_{1}{ }^{2} \sin ^{2}\left(\vartheta_{1}\right) \sigma_{\Delta \varphi 1}^{2}+V_{2}{ }^{2} \sin ^{2}\left(\vartheta_{2}\right) \sigma_{\Delta \varphi 2}^{2}+V_{3}{ }^{2} \sin ^{2}\left(\vartheta_{3}\right) \sigma_{\Delta \varphi 3}^{2} \\
\\
\sigma_{V}^{2}=V_{1}{ }^{2} \sin ^{2}\left(\vartheta_{1}\right) \sigma_{\varepsilon 1}^{2}+V_{2}{ }^{2} \sin ^{2}\left(\vartheta_{2}\right) \sigma_{\varepsilon 2}^{2}+V_{3}{ }^{2} \sin ^{2}\left(\vartheta_{3}\right) \sigma_{\varepsilon 3}^{2}+ \\
V_{1}{ }^{2} \cos ^{2}\left(\vartheta_{1}\right) \sigma_{\Delta \varphi 1}^{2}+V_{2}{ }^{2} \cos ^{2}\left(\vartheta_{2}\right) \sigma_{\Delta \varphi 2}^{2}+V_{3}{ }^{2} \cos ^{2}\left(\vartheta_{3}\right) \sigma_{\Delta \varphi 3}^{2} .
\end{array}
$$

Where:

$$
\begin{gathered}
U=\operatorname{Re}\left[\bar{V}_{R}\right] \\
V=\operatorname{Im}\left[\bar{V}_{R}\right]
\end{gathered}
$$

In light of (27) and (28), (14) can be rewritten as:

$$
\left|\bar{V}_{R}\right|=W=\sqrt{U^{2}+V^{2}} .
$$

If 3 LPVTs with the same accuracy class are assumed, hence $\sigma_{\varepsilon 1}^{2}=\sigma_{\varepsilon 2}^{2}=\sigma_{\varepsilon 3}^{2}=\sigma_{\varepsilon}^{2}$ and $\sigma_{\Delta \varphi 1}^{2}=\sigma_{\Delta \varphi 2}^{2}=\sigma_{\Delta \varphi 3}^{2}=\sigma_{\Delta \varphi}^{2},(25)$ and (26) turn into:

$$
\begin{aligned}
\sigma_{U}^{2}= & \sigma_{\varepsilon}^{2}\left(V_{1}{ }^{2} \cos ^{2}\left(\vartheta_{1}\right)+V_{2}{ }^{2} \cos ^{2}\left(\vartheta_{2}\right)+V_{3}{ }^{2} \cos ^{2}\left(\vartheta_{3}\right)\right)+ \\
& \sigma_{\Delta \varphi}^{2}\left(V_{1}{ }^{2} \sin ^{2}\left(\vartheta_{1}\right)+V_{2}{ }^{2} \sin ^{2}\left(\vartheta_{2}\right)+V_{3}{ }^{2} \sin ^{2}\left(\vartheta_{3}\right)\right) \\
\sigma_{V}^{2}= & \sigma_{\varepsilon}^{2}\left(V_{1}{ }^{2} \sin ^{2}\left(\vartheta_{1}\right)+V_{2}{ }^{2} \sin ^{2}\left(\vartheta_{2}\right)+V_{3}{ }^{2} \sin ^{2}\left(\vartheta_{3}\right)\right)+ \\
& \sigma_{\Delta \varphi}^{2}\left(V_{1}{ }^{2} \cos ^{2}\left(\vartheta_{1}\right)+V_{2}{ }^{2} \cos ^{2}\left(\vartheta_{2}\right)+V_{3}{ }^{2} \cos ^{2}\left(\vartheta_{3}\right)\right) .
\end{aligned}
$$

Hence:

where

$$
\begin{gathered}
\sigma_{U}^{2}=a \sigma_{\varepsilon}^{2}+b \sigma_{\Delta \varphi}^{2} \\
\sigma_{V}^{2}=b \sigma_{\varepsilon}^{2}+a \sigma_{\Delta \varphi}^{2}
\end{gathered}
$$

$$
\begin{gathered}
a=V_{1}{ }^{2} \cos ^{2}\left(\vartheta_{1}\right)+V_{2}{ }^{2} \cos ^{2}\left(\vartheta_{2}\right)+V_{3}{ }^{2} \cos ^{2}\left(\vartheta_{3}\right) \\
b=V_{1}{ }^{2} \sin ^{2}\left(\vartheta_{1}\right)+V_{2}{ }^{2} \sin ^{2}\left(\vartheta_{2}\right)+V_{3}{ }^{2} \sin ^{2}\left(\vartheta_{3}\right)
\end{gathered}
$$

are completely known to in-field operators.

From (32) and (33) it is clear that the variances of $U$ and $V$ are different, hence the Rayleigh distribution adopted in [9] cannot be applied anymore. Consequently, a different strategy is to be applied.

In light of (15) and (16), $U$ and $V$ are the sum of several random variables. Therefore, according to the Central Limit Theorem, they are two normal random variables $U\left(\mu_{U}, \sigma_{U}^{2}\right)$ and $V\left(\mu_{V}, \sigma_{V}^{2}\right)$, respectively. It is:

$$
\begin{aligned}
& \mu_{U}=V_{1} \cos \left(\vartheta_{1}\right)+V_{2} \cos \left(\vartheta_{2}\right)+V_{3} \cos \left(\vartheta_{3}\right) \\
& \mu_{V}=V_{1} \sin \left(\vartheta_{1}\right)+V_{2} \sin \left(\vartheta_{2}\right)+V_{3} \sin \left(\vartheta_{3}\right) .
\end{aligned}
$$

The squares $U^{2}$ and $V^{2}$ of $U$ and $V$ have a chi-square distribution $\left(\chi^{2}\right)$ with one degree of freedom [20,21]. Therefore, it is:

and

$$
\begin{aligned}
& \sigma_{U^{2}}^{2}=2\left(1+2\left(\frac{\mu_{U}}{\sigma_{U}}\right)^{2}\right) \sigma_{U}^{4} \\
& \sigma_{V^{2}}^{2}=2\left(1+2\left(\frac{\mu_{V}}{\sigma_{V}}\right)^{2}\right) \sigma_{V}^{4}
\end{aligned}
$$

$$
\begin{aligned}
& \mu_{U^{2}}=\sigma_{U}^{2}+\mu_{U}^{2} \\
& \mu_{V^{2}}=\sigma_{V}^{2}+\mu_{V}^{2}
\end{aligned}
$$

Where $\sigma_{U^{2}}^{2}, \mu_{U^{2}}$ and $\sigma_{V^{2}}^{2}, \mu_{V^{2}}$ are the variance and the mean value of the two random variables $U^{2}$ and $V^{2}$, respectively.

Finally, $W^{2}=U^{2}+V^{2}$ is the sum of two generic random variables, which variance $\sigma_{W^{2}}^{2}$ is, according to (24):

$$
\sigma_{W^{2}}^{2}=\sigma_{U^{2}}^{2}+\sigma_{V^{2}}^{2}+2 \operatorname{cov}\left(U^{2}, V^{2}\right) .
$$

and which mean $\mu_{W^{2}}$ is:

$$
\mu_{W^{2}}=\mu_{U^{2}}+\mu_{V^{2}}
$$

Eq. (42) is more genera than (24). In fact, the covariance term $\operatorname{cov}\left(U^{2}, V^{2}\right)$ has been added to consider also the case of non-independents variables. In the presented case, $U^{2}$ and $V^{2}$ are surely related. However, as it is confirmed in the result Section, neglecting their covariance does not affect the overall results. Of course, further studies can be performed to deep analyse such a behaviour.

The distribution associated to $W^{2}$ is still a chi-square one, which is a special case of the gamma distribution [20, 21]. According to $[22,23]$ and in light of the aforementioned results, $W=\sqrt{W^{2}}$ follows a Nakagami distribution with the shape and the spread parameters $m$ and $\Omega$, respectively:

$$
\begin{gathered}
m=\frac{\left(\mu_{W^{2}}\right)^{2}}{\sigma_{W^{2}}^{2}} \\
\Omega=\mu_{W^{2}}
\end{gathered}
$$

Considering that terms inside (44) and (45) are well-known from the previous steps, $\mu_{W}$ and $\sigma_{W}^{2}$ can be computed as:

$$
\begin{gathered}
\mu_{W}=\frac{\Gamma(m+1 / 2)}{\Gamma(m)} \sqrt{\frac{\Omega}{m}} \\
\sigma_{W}^{2}=\Omega\left[1-\frac{1}{m}\left(\frac{\Gamma(m+1 / 2)}{\Gamma(m)}\right)^{2}\right] .
\end{gathered}
$$


TABLE I. LIST OF THE PHASORS VALUES FOR THE 15 TESTS

\begin{tabular}{|c|c|c|c|c|c|c|c|}
\hline Test & $\begin{array}{c}\text { Acc. } \\
\text { class }\end{array}$ & $\boldsymbol{V}_{\mathbf{1}}[\mathbf{V}]$ & $\boldsymbol{V}_{\mathbf{2}}[\mathbf{V}]$ & $\boldsymbol{V}_{\mathbf{3}}[\mathbf{V}]$ & $\begin{array}{c}\boldsymbol{\vartheta}_{\mathbf{1}} \\
{\left[{ }^{\circ}\right]}\end{array}$ & $\begin{array}{c}\boldsymbol{\vartheta}_{\mathbf{2}} \\
{\left[{ }^{\circ}\right]}\end{array}$ & $\begin{array}{c}\boldsymbol{\vartheta}_{\mathbf{3}} \\
{\left[{ }^{\circ}\right]}\end{array}$ \\
\hline$\# 1$ & 0.1 & 11547 & 11547 & 11547 & 0 & -120 & 120 \\
\hline$\# 2$ & 0.1 & 12124 & 12124 & 11547 & 0 & -120 & 120 \\
\hline$\# 3$ & 0.1 & 12124 & 10392 & 11547 & 0 & -120 & 120 \\
\hline$\# 4$ & 0.1 & 11547 & 11547 & 11547 & 0 & -120 & 135 \\
\hline$\# 5$ & 0.1 & 12124 & 10392 & 11547 & 0 & -110 & 130 \\
\hline$\# 6$ & 0.2 & 11547 & 11547 & 11547 & 0 & -120 & 120 \\
\hline$\# 7$ & 0.2 & 12124 & 12124 & 11547 & 0 & -120 & 120 \\
\hline$\# 8$ & 0.2 & 12124 & 10392 & 11547 & 0 & -120 & 120 \\
\hline$\# 9$ & 0.2 & 11547 & 11547 & 11547 & 0 & -120 & 135 \\
\hline$\# 10$ & 0.2 & 12124 & 10392 & 11547 & 0 & -110 & 130 \\
\hline$\# 11$ & 0.5 & 11547 & 11547 & 11547 & 0 & -120 & 120 \\
\hline$\# 12$ & 0.5 & 12124 & 12124 & 11547 & 0 & -120 & 120 \\
\hline$\# 13$ & 0.5 & 12124 & 10392 & 11547 & 0 & -120 & 120 \\
\hline$\# 14$ & 0.5 & 11547 & 11547 & 11547 & 0 & -120 & 135 \\
\hline$\# 15$ & 0.5 & 12124 & 10392 & 11547 & 0 & -110 & 130 \\
\hline
\end{tabular}

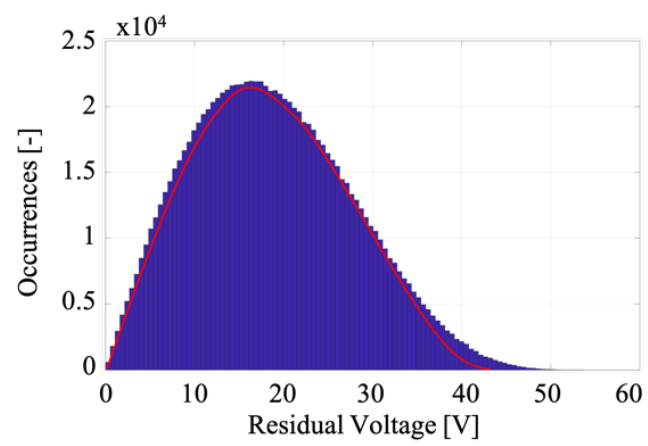

Fig. 2. PDF of $\left|\bar{V}_{R}\right|$ when three 0.1 class LPVTs are considered (case \#1)

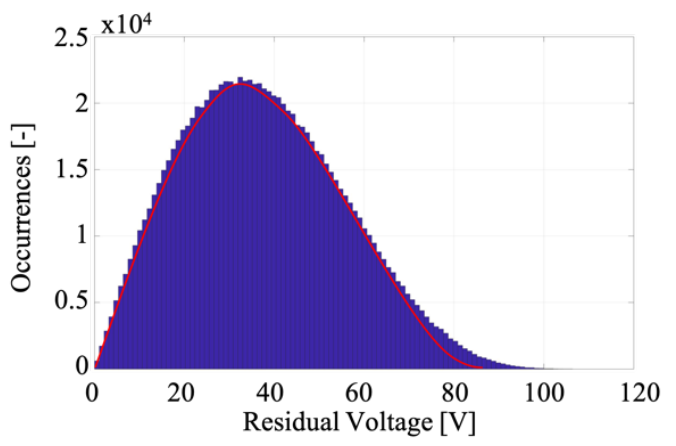

Fig. 3. PDF of $\left|\bar{V}_{R}\right|$ when three 0.2 class LPVTs are considered (case \#6)

$\Gamma(m)$ is the gamma function with $m$ degrees of freedom. These two expressions allow, at a glance, to determine the mean value and the variance of the residual voltage module $\left|\bar{V}_{R}\right|=W$ (see (29)). As a further comment, the term $\frac{\Gamma(m+1 / 2)}{\Gamma(m)}$ in both (46) and (47) is also known as the Pochhammer function. Such a function can be used in some softwares, instead of the Gamma one, to prevent computational issues that may occur when $m$ takes high values.

Summarizing, (46) and (47) allow to determine the mean value and variance of the residual voltage module, by simply measuring the 3 voltage phasors by means of three LPVTs with given accuracy class. It should be noted that, the provided

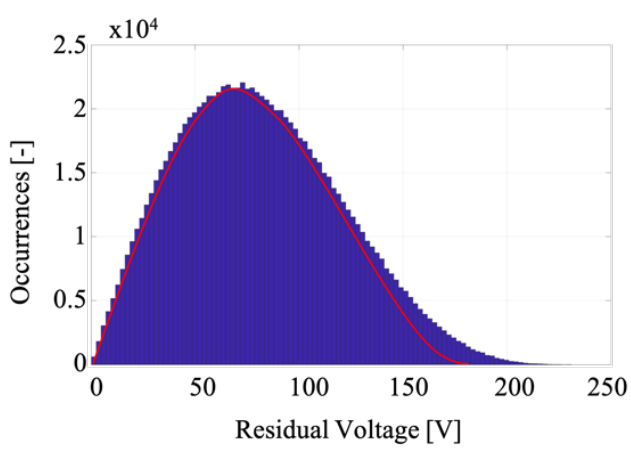

Fig. 4. PDF of $\left|\bar{V}_{R}\right|$ when three 0.5 class LPVTs are considered (case \#11)

expression can also be used even in the case of non-sinusoidal waveforms. As a matter of fact, a Fourier transform is usually applied to extract the phasor component at power frequency (50 $\mathrm{Hz}$ ), in the case of non-sinusoidal condition. Hence, the proposed expression application can be extended also to this case, by replacing the ratio and phase error of the LPVT with the corresponding ones derived by their propagation through the Fourier transform algorithm [24]. In particular, in presence of noise affecting the input voltage, the application of the Fourier transform allows to neglect the effect of the noise on the phasor estimation in all the practical situations. As a matter of fact, in [25] it was shown that already with a signal to noise ratio of 20 , the error due to the noise on the estimate of the signal components is a fraction of percent. Hence, by considering that a typical signal-to-noise ratio in power system is below $1 \%$, it results that the noise contribution to the overall uncertainty can be considered negligible.

\section{Monte Carlo results.}

Monte Carlo (MC) method is applied to validate (46) and (47). Usually, the LPVTs manufacturers do not provide information regarding the probability distribution of ratio and phase errors. Therefore, in accordance with $[12,13]$, this lack of knowledge leads to assume the 6 random variables in (15) and (16) as uniformly distributed within the intervals which limits are defined by the accuracy class specified by the LPVTs manufacturers. To assess the proposed expressions in actual conditions, LPVTs featuring $20 / \sqrt{3} \mathrm{kV}-3.25 / \sqrt{3} \mathrm{~V}$ have been considered. In particular, three different set of LPVTs have been simulated. In each set all of them present the same accuracy class: $0.1,0.2$ or 0.5 . For the three above sets, 5 combinations of three voltage phasors of a three-phase system have been tested. The values have been chosen according to the voltage limits provided by the EN 50160 [26] and are listed in Table I. Then, 1 million MC trials are computed to estimate the probability density function (PDF), the mean and the variance of $\left|\bar{V}_{R}\right|$. As for $\left|\bar{V}_{R}\right|$, Fig. 2, 3 and 4 show its PDFs in the case of test $\# 1$ and accuracy class $0.1,0.2$ and 0.5 , respectively. From these figures, it can be highlighted that the PDF shape is consistent with the Nakagami distribution (plotted in the graphs along with $\left|\bar{V}_{R}\right|$ ) adopted in the previous subsection to represent the $\left|\bar{V}_{R}\right|$. The same observation holds for all the other cases, which PDF are not reported for the sake of brevity.

Afterwards, mean value and variance of $\left|\bar{V}_{R}\right|$ resulting from MC are compared with the ones obtained by applying (46) and (47). Test results are reported in Table II, where the subscripts 
TABLE II. MEAN VALUE AND VARIANCE OF $\left|\bar{V}_{R}\right|$ FOR THE \#15 TESTS APPLYING THE MC METHOD AND THE PROPOSED EXPRESSION

\begin{tabular}{|c|c|c|c|c|c|}
\hline Test & $\begin{array}{c}\text { Acc. } \\
\text { Class }\end{array}$ & $\begin{array}{c}\mu_{M C} \\
{[\mathrm{~V}]}\end{array}$ & $\begin{array}{c}\sigma_{M C}^{2} \\
{\left[\mathrm{~V}^{2}\right]}\end{array}$ & $\begin{array}{c}\mu_{N} \\
{[\mathrm{~V}]}\end{array}$ & $\begin{array}{c}\sigma_{N}^{2} \\
{\left[\mathrm{~V}^{2}\right]}\end{array}$ \\
\hline$\# 1$ & 0.1 & 19 & 82 & 18 & 93 \\
\hline$\# 2$ & 0.1 & 577 & 234 & 577 & 232 \\
\hline$\# 3$ & 0.1 & 1528 & 214 & 1528 & 210 \\
\hline$\# 4$ & 0.1 & 3014 & 217 & 3014 & 213 \\
\hline$\# 5$ & 0.1 & 1471 & 196 & 1471 & 208 \\
\hline$\# 6$ & 0.2 & 38 & 328 & 37 & 372 \\
\hline$\# 7$ & 0.2 & 578 & 936 & 578 & 925 \\
\hline$\# 8$ & 0.2 & 1528 & 857 & 1528 & 838 \\
\hline$\# 9$ & 0.2 & 3015 & 867 & 3015 & 852 \\
\hline$\# 10$ & 0.2 & 1471 & 783 & 1471 & 833 \\
\hline$\# 11$ & 0.5 & 81 & 1561 & 80 & 1745 \\
\hline$\# 12$ & 0.5 & 581 & 4338 & 581 & 4268 \\
\hline$\# 13$ & 0.5 & 1529 & 3983 & 1529 & 3931 \\
\hline$\# 14$ & 0.5 & 3015 & 4072 & 3015 & 4032 \\
\hline$\# 15$ & 0.5 & 1472 & 3804 & 1472 & 3921 \\
\hline
\end{tabular}

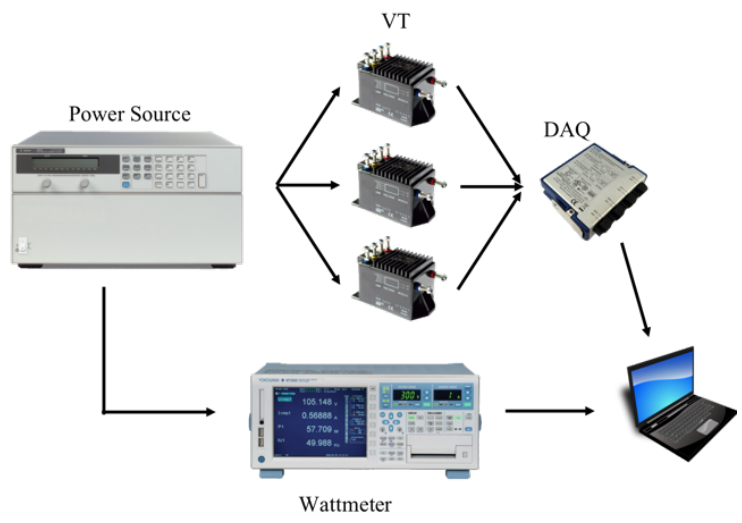

Fig. 5. Schematic of the measurement setup adopted for the residual voltage measurement

$\mathrm{MC}$ and $\mathrm{N}$ refer to the Monte Carlo and the analytical expression based on the Nakagami distribution, respectively. The comparison shows that, for each accuracy class, mean values and variances provided by (46) and (47) are full consistent and substantially equal to the ones obtained by the MC trials. This holds for all the performed tests (range $19-3000 \mathrm{~V}$, which contains all the actual MV residual voltage values). In other words, the developed expression may be conveniently adopted in whatever power network conditions and for whatever absolute value of the residual voltage. It only requires the knowledge of the measured voltages and the accuracy classes of the LPVTs installed in the network. As a further comment, as expected, the variance significantly increases with the accuracy class. Moreover, aside from case \#1, \#6 and \#11 (where the residual voltage is not greater than $0.7 \%$ of the rated voltage), the variance is substantially independent of the residual voltage value.

\section{EXPERIMENTAL SETUP}

An experimental setup has been designed to generate a programmable (in amplitude and phase) low-voltage 3-phase system and to evaluate the residual voltage module $\left|\bar{V}_{R}\right|$. The choice of a low-voltage (LV) system, instead of a mediumvoltage (MV) one, is due to the availability of programmable LV sources. This does not affect the assess of the proposed expressions, that can be used no matter the input voltage. The system, depicted in Fig. 5, consists of:

- Agilent 6834B AC programmable Power source, featuring maximum values of $300 \mathrm{Vrms}$ and $4500 \mathrm{VA}$, a frequency range $45 \div 5000 \mathrm{~Hz}$.

- Yokogawa WT3000 Wattmeter featuring $0.01 \%$ of reading $(\% \mathrm{R})+0.03 \%$ of range accuracy $(\% \mathrm{FS})$ on the voltage measurement and 1 digit on the phase measurement. It acts as a reference for $\left|\bar{V}_{R}\right|$ measurement.

- 3 VT LEM CV-3-1000, featuring conversion ratio $10000 \mathrm{~V} / 10 \mathrm{~V}$, rated voltage of $700 \mathrm{~V}$ and 0.2 accuracy class. It introduces a negligible phase delay according to its datasheet. As for the noise introduced by the VT, the datasheet does not report any value, hence it has been considered negligible in terms of uncertainty computation.

- A 24-bit NI 9239 Data AcQuisition board (DAQ), and a Personal Computer (PC). Its related uncertainty has been neglected in the measurement chain due to its very little contribute with respect to the others measurement chain components $(0.03 \%$ of reading, $0.008 \%$ of range). In particular, its accuracy parameters are at least one order of magnitude lower than the LPVTs ones. Furthermore, the DAQ has an input noise of $70 \mu \mathrm{V}$, which is approximately $10^{-4}$ lower than the secondary outputs obtained (about $1 \mathrm{~V}$ ) as detailed in the following Sections. Such effect is negligible compared to the contribution of the LPVT, which is at least one order of magnitude greater.

In a nutshell, the 3-phase Power Source feeds both the Wattmeter and the 3 LPVTs. Then, the voltages needed for the residual voltage computation are acquired via LabView from the Wattmeter and via DAQ from the 3 VTs. Afterwards, data are collected and processed through a PC.

\section{EXPERIMENTAL TESTS \& RESUlts}

In this Section tests and results of the residual voltage measurement in actual condition are presented. Twenty different tests have been performed. In each of them amplitude and phase of the 3 phasors have been varied within the limits defined by the Standard EN 50160 [26]. Hence, the resulting \#20 test conditions, that have been used has input quantities, are listed in Table III whereas Table IV reports the relevant results. For each test, the absolute value of the residual voltage $\left|\bar{V}_{R}\right|$ has been measured 100 times by both the Wattmeter and the DAQ. Thus, such measurements have been used to compute $\left|\bar{V}_{R}\right|$ mean value and standard deviation of the mean.

Table IV contains:

- $\left|\bar{V}_{R}\right|$ mean value $\left|\bar{V}_{R}\right|_{W}$ and its combined uncertainty $u_{W}$ for the measurements performed with the wattmeter. The latter has been computed as: 
TABLE III. LIST OF THE PHASORS VALUES FOR EACH OF THE 20 TESTS PERFORMED

\begin{tabular}{|c|c|c|c|c|c|c|}
\hline Test & $\begin{array}{c}\boldsymbol{V}_{\mathbf{1}} \\
{[\mathbf{V}]}\end{array}$ & $\boldsymbol{V}_{\mathbf{2}}[\mathbf{V}]$ & $\boldsymbol{V}_{\mathbf{3}}[\mathbf{V}]$ & $\begin{array}{c}\boldsymbol{\vartheta}_{\mathbf{1}} \\
{\left[{ }^{\circ}\right]}\end{array}$ & $\boldsymbol{\vartheta}_{\mathbf{2}}\left[^{\circ}\right]$ & $\boldsymbol{\vartheta}_{\mathbf{3}}\left[^{\circ}\right]$ \\
\hline$\# 1$ & 230 & 230 & 230 & 0 & -120 & 120 \\
\hline$\# 2$ & 207 & 230 & 230 & 0 & -120 & 120 \\
\hline$\# 3$ & 218.5 & 230 & 230 & 0 & -120 & 120 \\
\hline$\# 4$ & 253 & 230 & 230 & 0 & -120 & 120 \\
\hline$\# 5$ & 241.5 & 230 & 230 & 0 & -120 & 120 \\
\hline$\# 6$ & 207 & 218.5 & 230 & 0 & -120 & 120 \\
\hline$\# 7$ & 207 & 207 & 230 & 0 & -120 & 120 \\
\hline$\# 8$ & 207 & 241.5 & 230 & 0 & -120 & 120 \\
\hline$\# 9$ & 207 & 253 & 230 & 0 & -120 & 120 \\
\hline$\# 10$ & 218.5 & 218.5 & 230 & 0 & -120 & 120 \\
\hline$\# 11$ & 218.5 & 241.5 & 230 & 0 & -120 & 120 \\
\hline$\# 12$ & 218.5 & 253 & 230 & 0 & -120 & 120 \\
\hline$\# 13$ & 230 & 230 & 0 & 0 & -120 & 0 \\
\hline$\# 14$ & 230 & 230 & 230 & 0 & -120 & 130 \\
\hline$\# 15$ & 230 & 230 & 230 & 0 & -120 & 125 \\
\hline$\# 16$ & 230 & 230 & 230 & 0 & -120 & 115 \\
\hline$\# 17$ & 230 & 230 & 230 & 0 & -120 & 110 \\
\hline$\# 18$ & 230 & 230 & 230 & 0 & -120 & 121 \\
\hline$\# 19$ & 230 & 230 & 230 & 0 & -121 & 121 \\
\hline$\# 20$ & 230 & 230 & 218.5 & 0 & -120 & 121 \\
\hline & & & & & & \\
\hline
\end{tabular}

TABLE IV. RESIDUAL VOLTAGE MEASUREMENT RESULTS COMPARISON BETWEEN SIMULATIONS AND ACTUAL TESTS

\begin{tabular}{|c|c|c|c|c|c|c|}
\hline Test & $\begin{array}{c}\left|\bar{V}_{R}\right|_{W} \\
{[\mathrm{~V}]}\end{array}$ & $u_{W}[\mathrm{~V}]$ & $\left|\bar{V}_{R}\right|_{D A Q}[\mathrm{~V}]$ & $\begin{array}{c}\sigma_{D A Q} \\
{[\mathrm{~V}]}\end{array}$ & $\begin{array}{c}\left|\bar{V}_{R}\right|_{N} \\
{[\mathrm{~V}]}\end{array}$ & $\begin{array}{c}u_{N} \\
{[\mathrm{~V}} \\
]\end{array}$ \\
\hline$\# 1$ & 0.22 & $5 \cdot 10^{-2}$ & 0.6542 & $3 \cdot 10^{-4}$ & 1.0 & 0.5 \\
\hline$\# 2$ & 22.78 & $8 \cdot 10^{-2}$ & 22.6130 & $1 \cdot 10^{-4}$ & 22.6 & 0.6 \\
\hline$\# 3$ & 11.23 & $7 \cdot 10^{-2}$ & 11.15434 & $4 \cdot 10^{-5}$ & 11.2 & 0.6 \\
\hline$\# 4$ & 23.22 & $7 \cdot 10^{-2}$ & 23.43270 & $3 \cdot 10^{-5}$ & 23.4 & 0.6 \\
\hline$\# 5$ & 11.78 & $7 \cdot 10^{-2}$ & 11.85376 & $4 \cdot 10^{-5}$ & 11.9 & 0.6 \\
\hline$\# 6$ & 19.54 & $8 \cdot 10^{-2}$ & 19.31514 & $3 \cdot 10^{-5}$ & 19.3 & 0.6 \\
\hline$\# 7$ & 22.59 & $7 \cdot 10^{-2}$ & 22.33561 & $4 \cdot 10^{-5}$ & 22.3 & 0.5 \\
\hline$\# 8$ & 30.29 & $7 \cdot 10^{-2}$ & 30.26731 & $3 \cdot 10^{-5}$ & 30.3 & 0.6 \\
\hline$\# 9$ & 39.75 & $6 \cdot 10^{-2}$ & 39.80380 & $4 \cdot 10^{-5}$ & 39.8 & 0.6 \\
\hline$\# 10$ & 11.07 & $7 \cdot 10^{-2}$ & 10.8727 & $3 \cdot 10^{-4}$ & 10.9 & 0.6 \\
\hline$\# 11$ & 19.80 & $6 \cdot 10^{-2}$ & 19.91989 & $4 \cdot 10^{-5}$ & 19.9 & 0.6 \\
\hline$\# 12$ & 30.48 & $6 \cdot 10^{-2}$ & 30.54182 & $5 \cdot 10^{-5}$ & 30.5 & 0.6 \\
\hline$\# 13$ & 230.21 & $8 \cdot 10^{-2}$ & 230.23121 & $3 \cdot 10^{-5}$ & 230.2 & 0.5 \\
\hline$\# 14$ & 39.99 & $6 \cdot 10^{-2}$ & 40.02377 & $5 \cdot 10^{-5}$ & 40.0 & 0.6 \\
\hline$\# 15$ & 20.04 & $6 \cdot 10^{-2}$ & 20.0049 & $1 \cdot 10^{-4}$ & 20.0 & 0.6 \\
\hline$\# 16$ & 20.19 & $6 \cdot 10^{-2}$ & 20.09044 & $5 \cdot 10^{-5}$ & 20.1 & 0.6 \\
\hline$\# 17$ & 40.19 & $6 \cdot 10^{-2}$ & 40.15524 & $5 \cdot 10^{-5}$ & 40.2 & 0.6 \\
\hline$\# 18$ & 3.93 & $6 \cdot 10^{-2}$ & 4.03867 & $5 \cdot 10^{-5}$ & 4.1 & 0.6 \\
\hline$\# 19$ & 6.65 & $7 \cdot 10^{-2}$ & 6.6032 & $1 \cdot 10^{-4}$ & 6.6 & 0.6 \\
\hline$\# 20$ & 12.61 & $7 \cdot 10^{-2}$ & 12.69038 & $4 \cdot 10^{-5}$ & 12.7 & 0.6 \\
\hline
\end{tabular}

$$
u_{W}=\sqrt{\sigma_{W a}^{2}+\sigma_{W b}^{2}}
$$

where $\sigma_{W a}$ is the standard deviation of the mean and $\sigma_{W b}$ is the standard uncertainty evaluated with type B method as explained in [12, 13], starting from the wattmeter nominal accuracy specifications reported in Section IV;
TABLE V $\quad 95 \%$-CONFIDENCE

INTERVAL LIMITS FOR THE MEAN VALUES $\left|\bar{V}_{R}\right|_{D A Q}$ AND $\left|\bar{V}_{R}\right|_{N}$

\begin{tabular}{|c|c|c|c|c|}
\hline \multirow{2}{*}{ Test } & \multicolumn{2}{|c|}{$\left|\bar{V}_{R}\right|_{W}$} & \multicolumn{2}{|c|}{$\left|\bar{V}_{R}\right|_{N}$} \\
\hline & $-L[V]$ & $+L[V]$ & $-L[V]$ & $+L[V]$ \\
\hline$\# 1$ & 0.11 & 0.31 & 0.0 & 1.9 \\
\hline$\# 2$ & 22.63 & 22.93 & 21.5 & 23.8 \\
\hline$\# 3$ & 11.08 & 11.37 & 10.0 & 12.3 \\
\hline$\# 4$ & 23.07 & 23.37 & 22.2 & 24.6 \\
\hline$\# 5$ & 11.63 & 11.93 & 10.7 & 13.1 \\
\hline$\# 6$ & 19.39 & 19.69 & 18.2 & 20.4 \\
\hline$\# 7$ & 22.45 & 22.73 & 21.3 & 23.4 \\
\hline$\# 8$ & 30.16 & 30.43 & 29.1 & 31.4 \\
\hline$\# 9$ & 39.62 & 39.87 & 38.6 & 41.0 \\
\hline$\# 10$ & 10.92 & 11.21 & 9.8 & 12.0 \\
\hline$\# 11$ & 19.67 & 19.93 & 18.8 & 21.1 \\
\hline$\# 12$ & 30.35 & 30.61 & 29.4 & 31.7 \\
\hline$\# 13$ & 230.04 & 230.38 & 229.3 & 231.2 \\
\hline$\# 14$ & 39.86 & 40.11 & 38.9 & 41.2 \\
\hline$\# 15$ & 19.92 & 20.17 & 18.8 & 21.2 \\
\hline$\# 16$ & 20.07 & 20.32 & 18.9 & 21.3 \\
\hline$\# 17$ & 40.06 & 40.31 & 39.0 & 41.3 \\
\hline$\# 18$ & 3.82 & 4.05 & 2.9 & 5.2 \\
\hline$\# 19$ & 6.51 & 6.80 & 5.5 & 7.8 \\
\hline$\# 20$ & 12.47 & 12.75 & 11.5 & 13.9 \\
\hline
\end{tabular}

- $\quad\left|\bar{V}_{R}\right|$ mean value $\left|\bar{V}_{R}\right|_{D A Q}$ and standard deviation of the mean $\sigma_{D A Q}$ for the measurements performed with the 3 LPVTs and acquired by the DAQ. Such test wants to represent an actual condition where typical LPVTs are adopted. In $\sigma_{D A Q}$ evaluation, the contribution due to the data acquisition system has been neglected has explained before.

- $\left|\bar{V}_{R}\right|$ mean value $\left|\bar{V}_{R}\right|_{N}$ and combined uncertainty $u_{N}$ obtained starting from (46) and (47). The test consisted in using the three voltage phasors, measured with the LPVTs, as input for the proposed expression along with their accuracy class parameters. The $u_{N}$ has been computed has:

$$
u_{N}=\sqrt{\sigma_{D A Q}^{2}+\sigma_{N}^{2}}
$$

where $\sigma_{N}^{2}$ is the variance obtained from (47).

From Table IV, several comments arise. By comparing the results from the wattmeter measurements with the ones obtained from the proposed expression, it can be confirmed the latter efficiency. In detail, to assess the obtained results by comparing them with the reference ones obtained with the wattmeter, limits $( \pm \mathrm{L})$ of $95 \%$-confidence interval have been calculated for $\left|\bar{V}_{R}\right|_{W}$ and $\left|\bar{V}_{R}\right|_{N}$ mean values. Such intervals limits are listed, for all the tests, in Table V. Both intervals have been computed by considering a coverage factor $\mathrm{k}=2$. Such assumption is justified also for the Nakagami distribution as explained at the end of the Section. As it emerges from Table V, for each test the two different intervals superimpose one each other, confirming the goodness and applicability of the simple method presented. For the sake of clarity, Fig. 6 shows, for the cases \#2, \#3 and \#4 a graphical representation of the mean values along with their confidence intervals. For all the cases, the two intervals, referred as $\mathrm{N}$ and $\mathrm{W}$ to be consistent with Table $\mathrm{V}$, have been graphed one next to the other to better highlight the superimposition. 


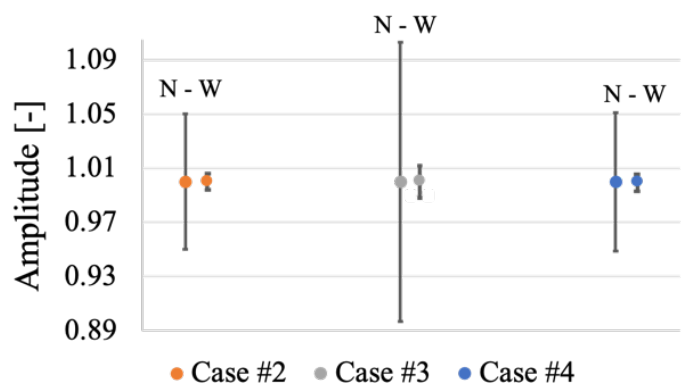

Fig. 6. Graphical representation of the measurements and their $95 \%$ confidence intervals, in the case of tests \#2, \#3 and \#4

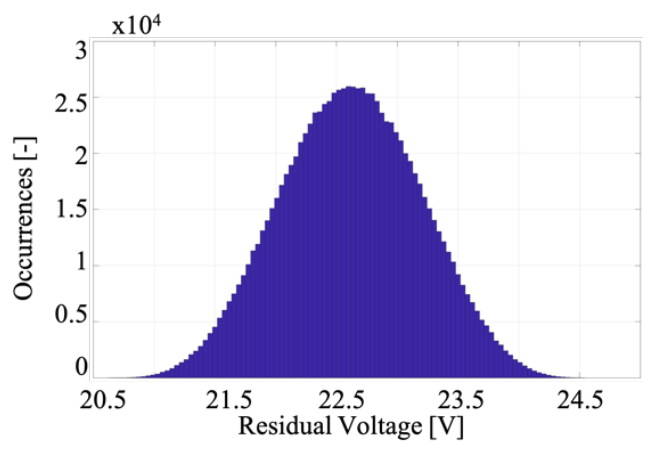

Fig. 7. PDF of $\left|\bar{V}_{R}\right|_{N}$ for case \#2

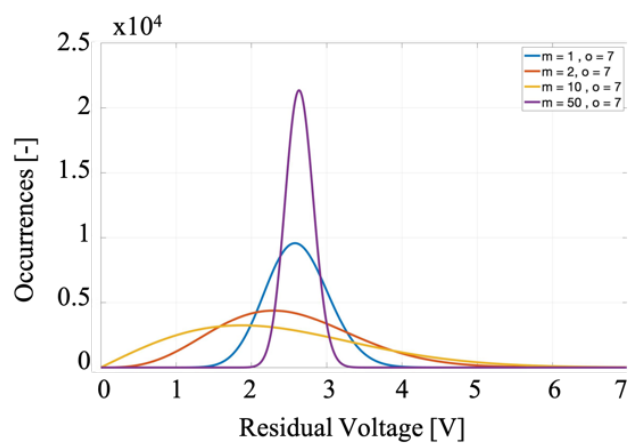

Fig. 8. PDF of a generic Nakagami distribution for several $m$ and $\omega$ (o) parameters.

The high non-linearity of the residual voltage module expression (29) leads to a non-symmetrical probability density function associated to the $\left|\bar{V}_{R}\right|$ random variable. As a matter of fact, the Nakagami distribution may exhibit a non-symmetrical shape for particular values of its parameters. The asymmetry is caused by the module which converts positive and negative real and imaginary terms of $\bar{V}_{R}$ into always positive terms. This results in a shift of the $\left|\bar{V}_{R}\right|$ mean value from the measured value of $\left|\bar{V}_{R}\right|$. Moreover, this effect is considerable when the latter is close to zero (case \#1), while it becomes negligible as far as it increases. Such a conclusion can be verified with two examples. They both deal with the case \#1 where a balanced three-phase system is considered. Hence, the $\left|\bar{V}_{R}\right|$ is ideally zero. The first example refers to Table II, where the proposed expression is compared to the MC method. As it emerges from the $\mu_{M C}$ and $\mu_{N}$ values, they largely differ from the theoretical null one. The reason is explainable by the effect of the ratio and phase errors, treated as random variables with zero mean. This correctly leads to the shift of $\left|\bar{V}_{R}\right|$ mean value as detailed before.

The second example refers instead to case \#1 in Table IV. A little discrepancy can be noted between $\left|\bar{V}_{R}\right|_{D A Q}$ and $\left|\bar{V}_{R}\right|_{N}$, although they are computed starting from the same voltage phasors. The only difference from the previous example is that it refers to an actual measurement and not to a simulation.

As for the $\left|\bar{V}_{R}\right|_{N}$ distribution, it can be stated that it tends to a normal distribution (Fig. 7 shows the case \#2 PDF of $\left|\bar{V}_{R}\right|_{N}$ ). This holds for all the tests which means are far higher than zero. This occurs for all of them except for case \#1 (as confirmed by Fig. 2, 3 and 4). As a matter of fact, high mean values turn into an $m>1$ parameter inside (46) and (47). Therefore, in a balanced three-phase system, which feature $m=1$, the effect is a probability density function far from being a normal one. Fig. 8 confirms the previous statement showing the Nakagami distribution for several $m$ values. In light of the aforementioned, the $95 \%$-confidence intervals, previously described, have been computed assuming a symmetric distribution.

\section{CONCLUSIONS}

The introduction of the LPVT technology has allowed to implement many new operating and measurement functions due to their better performance with respect to inductive ITs (bandwidth, accuracy, linearity, etc.). However, one critical value, still widely used for the diagnostic and the operation of the power networks, namely the residual voltage, requires that LPVTs feature higher accuracies with respect to those of ITs with open triangle. The correlation of the accuracy class of LPVTs to the uncertainty affecting the residual voltage is not straightforward. However, this relationship is strongly demanded by practitioners and all people involved in network design and operation.

To this end, the presented paper extended a study began in a companion paper where an expression for the correlation between the LPIT accuracy class and the residual voltage uncertainty was presented. In that work a symmetrical 3-phase system was studied. In this paper an expression for a general 3phase system has been proposed by authors.

The aim of this study has been to put all such operators in a condition to simply evaluate and know which is the expected uncertainty affecting the residual voltage when employing LPVTs with a given accuracy class.

To this purpose, a simplified expression based on the Nakagami distribution has been derived. Results demonstrated the effectiveness of this assumption in whatever power network condition. Simulation results have been also confirmed by the measurements performed in an actual 3-phase system deployed in the laboratory. In light of the aforementioned, the proposed expression can become a simple and common way to predict the uncertainty related to the residual voltage measurement in all the actual situations and with an high accuracy.

\section{REFERENCES}

[1] A. Angioni, J. Shang, F. Ponci, A. Monti, "Real-time monitoring of distribution systems based on state estimation", IEEE Transaction on Instrumentation and Measurements, vol. 65, no. 10, pp. 2234-2243, 2016.

[2] IEC 61850-9-2:2011, "Communication networks and systems for power utility automation - Part 9-2: Specific communication service mapping (SCSM) - Sampled values over ISO/IEC 8802-3", International Standardization Organization, Geneva, Switzerland, 2008. 
[3] A. Mingotti, L. Peretto, R. Tinarelli, A. Angioni, A. Monti and F. Ponci, "Calibration of Synchronized Measurement System: from the Instrument Transformer to the PMU", IEEE workshop on Applied Measurements for Power Systems, AMPS, Bologna, Sep. 2018.

[4] IEC 61869-1:2011, "Instrument transformers - Part 1: General requirements", International Standardization Organization, Geneva, Switzerland, 2011.

[5] IEC 61869-2:2011, "Instrument transformers - Part 2: Additional requirements for current transformers", International Standardization Organization, Geneva, Switzerland, 2011.

[6] IEC 61869-3:2011, "Instrument transformers - Part 3: Additional requirements for inductive voltage transformers", International Standardization Organization, Geneva, Switzerland.

[7] A. Mingotti, L. Peretto, R. Tinarelli, L. Bartolomei and P. Rinaldi, "Accuracy Verification of PLL-Based Acquisition System for Low-Cost Applications", IEEE workshop on Applied Measurements for Power Systems, AMPS, Bologna, Sep. 2018.

[8] IEC 61869-13:2016, "Instrument transformers - Part 13: Stand Alone Mergin Unit", International Standardization Organization, Geneva, Switzerland.

[9] Sepam Series 80 - Protective Relays, Instruction Bulletin 63230-216230B1, Schneider Electric, 2007.

[10] A. Mingotti, L. Peretto, R. Tinarelli, F. Mauri, I. Gentilini, "Assessment of Metrological Characteristics of Calibration Systems for Accuracy vs. Temperature Verification of Voltage Transformers", IEEE workshop on Applied Measurements for Power Systems, AMPS, Liverpool, Sep. 2022, 2017.

[11] A. Mingotti, L. Peretto, R. Tinarelli, "Low Power Voltage Transformer Accuracy Class Effects on the Residual Voltage Measurement", Proc. of IEEE I2MTC, Houston, May 2018.

[12] ISO/IEC Guide 98-3:2008, "Uncertainty of measurement --Part 3: Guide to the expression of uncertainty in measurement (GUM:1995)", International Standardization Organization, Geneva, Switzerland, 2008.

[13] ISO/IEC Guide 98-3/Suppl.1:2008, "Evaluation of measurement data Supplement 1 to the "Guide to the expression of uncertainty in measurement" - Propagation of distributions using a Monte Carlo method", International Standardization Organization, Geneva, Switzerland 2008
[14] IEC 61869-11:2017, “Instrument transformers - Part 2: Additional requirements for current transformers", International Standardization Organization, Geneva, Switzerland, in press.

[15] IEC 61869-105, "Uncertainty in the calibration of Instrument Transformers", under preparation by IEC TC38/WG55.

[16] G. Crotti, D. Gallo, D. Giordano, C. Landi, M. Luiso, C. Cherbaucich, P. Mazza "Low cost measurement equipment for the accurate calibration of voltage and current transducers", IEEE International Instrumentation and Measurement Technology Conference, Montevideo, May, 2004.

[17] IEC 60050:2017, "International Electrotechnical Vocabulary (IEV)", 2017.

[18] A. Mingotti, L. Peretto, R. Tinarelli, K. Yigit, "Simplified Approach to Evaluate the Combined Uncertainty in Measurement Instruments for Power Systems", IEEE Transactions on Instrumentation and Measurement, vol. 66, n. 9, pp 2258-2265, 2017.

[19] M. Asprou; E. Kyriakides; M. Albu, "The Effect of Variable Weights in a WLS State Estimator Considering Instrument Transformer Uncertainties" IEEE Transactions on Instrumentation and Measurement, vol. 66, n. 9, pp 2258-2265, 2017.

[20] M. Evans, N. Hastings, B. Peacock, "Chi Distribution." $§ 8.3$ in Statistical Distributions, 3rd ed. New York: Wiley, p. 57, 2000

[21] N. Johnson, S. Kotz, Balakrishnan, N. Continuous Univariate Distributions, Vol. 1, 2nd ed. Boston, MA: Houghton Mifflin, 1994.

[22] Laurenson, Dave (1994). "Nakagami Distribution". Indoor Radio Channel Propagation Modelling by Ray Tracing Techniques. Retrieved 2007-08-04.

[23] J. F. Paris, "Nakagami-q (Hoyt) distribution function with applications", IEEE Electronic Letters, vol. 45, no. 4, pp. 210-211, Feb. 2009.

[24] G. Betta, C. Liguori, A. Pietrosanto, "Propagation of uncertainty in a discrete Fourier transform algorithm", Measurement, vol. 27, Issue 4, pp 231-239, 2000.

[25] J. F. Prewitt, "Amplitude bias in the Fourier transforms of noisy signals", IEEE Transactions on Antennas and Propagation, vol. 26, no. 5, pp 730731, Sep. 1978.

[26] EN 50160:2011, "Voltage characteristics of electricity supplied by public electricity networks", European committee for standardization, Brussels, 2017. 